\title{
Glycemic effect of pancreatic preproglucagon in mouse sleeve gastrectomy
}

\author{
Ki-Suk Kim, Chelsea R. Hutch, Landon Wood, Irwin J. Magrisso, Randy J. Seeley, \\ and Darleen A. Sandoval
}

Department of Surgery, University of Michigan, Ann Arbor, Michigan, USA.

\begin{abstract}
Intestinally derived glucagon-like peptide-1 (GLP-1), encoded by the preproglucagon (Gcg) gene, is believed to function as an incretin. However, our previous work questioned this dogma and demonstrated that pancreatic peptides rather than intestinal Gcg peptides, including GLP-1, are a primary regulator of glucose homeostasis in normal mice. The objective of these experiments was to determine whether changes in nutrition or alteration of gut hormone secretion by bariatric surgery would result in a larger role for intestinal CLP-1 in the regulation of insulin secretion and glucose homeostasis. Multiple transgenic models, including mouse models with intestine- or pancreas tissue-specific Gcg expression and a whole-body Gcg-null mouse model, were generated to study the role of organ-specific GLP-1 production on glucose homeostasis under dietary-induced obesity and after weight loss from bariatric surgery (vertical sleeve gastrectomy; VSG). Our findings indicated that the intestine is a major source of circulating GLP-1 after various nutrient and surgical stimuli. However, even with the 4-fold increase in intestinally derived GLP-1 with VSG, it is pancreatic peptides, not intestinal Gcg peptides, that are necessary for surgery-induced improvements in glucose homeostasis.
\end{abstract}

Conflict of interest: RJS has received research support from Ethicon Endo-Surgery, Zafgen, Novo Nordisk, Kallyope, and Medlmmune. RJS has served on scientific advisory boards for Ethicon Endo-Surgery, Novo Nordisk, Sanofi, Janssen, Kallyope, Scohia, and Ironwood Pharma. RJS is a stakeholder of Zafgen. DAS has received research support from Zafgen, Novo Nordisk, and Boehringer Ingelheim.

Copyright: (ㄷ) 2019, American Society for Clinical Investigation.

Submitted: April 9, 2019 Accepted: September 18, 2019 Published: September 24, 2019.

Reference information: JCI Insight. 2019;4(20):e129452. https://doi.org/10.1172/jci. insight.129452.

\section{Introduction}

Insulin responses to the same dose of glucose are greater during oral delivery compared with i.v. delivery (1). The dominant explanation for this "incretin effect" involves the postprandial secretion of peptide hormones from the gut that act on pancreatic $\beta$ cells to stimulate insulin secretion. Much of the subsequent literature has sought to identify the role of specific peptide hormones and their relative contribution to the incretin effect $(2,3)$. A time span of 2 decades went by before 2 particular gut peptides were identified that fit the definition of an incretin hormone: gastric inhibitory polypeptide (GIP) (4) and glucagon-like peptide-1 (GLP-1) (5). Studies demonstrating that i.v. administered GLP-1 stimulated insulin secretion in a glucose-dependent manner in humans (6) and pigs (7) supported an important role for this gut hormone as an incretin. Although GIP was found to be less effective in lowering glucose levels in patients with type 2 diabetes mellitus (T2DM) (8), GLP-1 receptor (GLP-1R) agonists and drugs that increase the half-life of endogenous GLP-1 have been successfully developed for the treatment of T2DM.

Whereas drugs that stimulate endogenous intestinal GLP-1 secretion are also in the pipeline for the treatment of T2DM $(9,10)$, physically manipulating the gastrointestinal (GI) tract with bariatric surgery clearly increases endogenous circulating GLP-1. Vertical sleeve gastrectomy (VSG), which is now the most commonly performed bariatric surgery worldwide $(11,12)$, increases postprandial GLP-1 levels several-fold in humans and rodents (13). Because GLP-1 possesses both incretin and anorectic effects (14), the postsurgical elevation has been implicated as a mechanism underpinning VSG-induced weight loss and improvements in glucose tolerance (reviewed in ref. 15).

The effort to understand the role of GLP-1 on VSG outcomes has solely focused on intestinal production. However, GLP-1 is not expressed only in the intestine and a discrete region of the hindbrain (nucleus of the solitary tract) (16); it is also expressed in pancreatic a cells (17). Recently, we demonstrated that preproglucagon products from the pancreas, but not from the intestine, are important in the regulation of normal glucose homeostasis (18). These findings challenge the prevailing hypothesis that GLP-1 from the enteroendocrine L cells is the key source of GLP-1 necessary for regulation of glucose homeostasis. Here, 


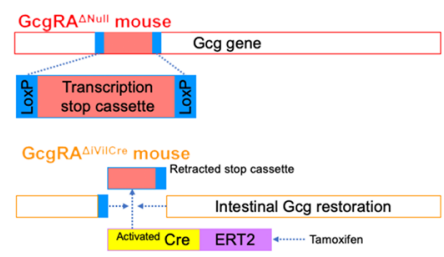

B

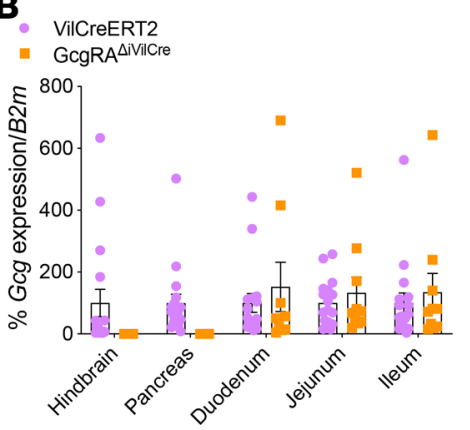

C
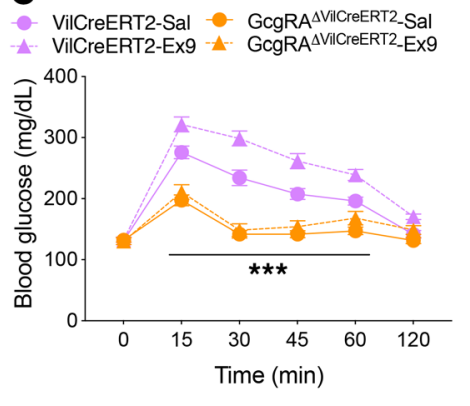

D VilCreERT2 - GcgRA VilCreERT2

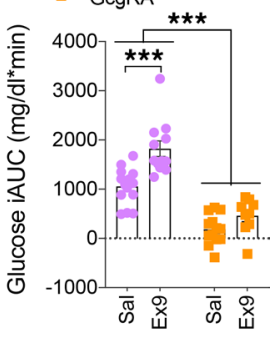

Figure 1. Tamoxifen-inducible intestinal Gcg reactivation in mice. (A) A brief schematic of the generation of the GcgRA ${ }^{\Delta V i l C r e E R T 2}$ mice. Mating of tamoxifen-inducible Villin-Cre mouse (VilCreERT2) with Gcg-null mouse (GcgRA ${ }^{\Delta N u l l}$ ) generates GcgRA ${ }^{\Delta V i l c r e E R T 2}$ offspring with intestinal Gcg reactivation. (B) Gcg gene expression from the hindbrain, pancreas, duodenum, jejunum, and ileum of GcgRA ${ }^{\Delta V i l c r e E R T 2}$ mice was not detectable in the hindbrain and pancreas but was at similar levels in all 3 sections of the small intestine compared with VilCreERT2 littermate controls. (C) Glucose response to an oral glucose load and an i.p. injection of saline (Sal) or exendin 9-39 (Ex9); 3-way ANOVA with Tukey post hoc; $P<$ 0.001 for Cre-Sal versus Cre-Ex9, but not significant for RA-Sal versus RA-Ex9 (genotype $\times$ drug); ${ }^{* *} P<0.001$ for Cre versus RA in both drug-treated groups (time $\times$ genotype). (D) Glucose incremental area under the curve (iAUC) during the oral glucose tolerance test (OCTT); 2-way ANOVA with Tukey post hoc; ${ }^{* *} P<0.001$ (genotype $\times$ drug). All data were obtained from cohort 1 , each animal was only studied once per condition, and data are represented as Mean \pm SEM. VilCreERT2 ( $n=17)$; GcgRA ${ }^{\Delta \text { VilCreERT2 }}(n=10)$.

we used a variety of novel mouse models to investigate 2 key questions: (a) what is the source, intestinal versus pancreatic, of circulating GLP-1 after various acute nutrient stimuli, chronic high-fat diet (HFD), and VSG? And (b) does increasing the secretion of gut-derived GLP-1 to supraphysiological levels reveal a larger role for this source of GLP-1 in regulation of glucose homeostasis?

\section{Results}

Intestinal GLP-1 is not insulinotropic. Plasma GLP-1 increases in response to consumption of fats, carbohydrates, and protein (19) and this effect is thought to be through multiple nutrient-sensing mechanisms within the intestine (20). To study the impact of equicaloric doses of macronutrients on intestinal GLP-1 and insulin levels, we crossed a previously validated mouse model (GcgRA $\left.{ }^{\Delta \mathrm{Null}}\right)$ that has a loxP-flanked transcriptional blocking cassette inserted into the Gcg gene (18) with a tamoxifen-inducible Villin-CreERT2 promoter mouse (VilCreERT2 mouse; ref. 21). This procedure allowed us to reactivate the normal intestinal expression of Gcg in adulthood (GcgRA $^{\Delta \mathrm{ViCreERT2}}$ ) (Figure 1A). We found that Gcg expression was specifically restored in the intestine but not in the hindbrain or pancreas in these mice (Figure 1B). Our previous work found that a developmental reactivation of intestinal Gcg did not restore the ability of exendin 9-39 (Ex9), a potent GLP-1R antagonist, to impair glucose tolerance (18). To validate these findings in the inducible model, Ex9, or saline (Sal) was injected 15 minutes before a glucose gavage (Figure $1, \mathrm{C}$ and D). Similar to our previous finding (18), glucose levels were significantly higher after Ex9 in control VilCreERT2 mice but had no effect on glucose levels in the GcgRA ${ }^{\Delta V i C r e E R T 2}$ mice. These data indicate intestinally secreted GLP-1 does not regulate glucose tolerance in mice under these conditions and replicates our previous data. We then measured GLP-1 and insulin responses to various macronutrients in the VilCreERT2 versus GcgRA ${ }^{\Delta V i l C r e E R T 2}$ mouse models. Oral administration of glucose, peptone, and intralipid at equicaloric doses all significantly increased intestinal GLP-1 levels to approximately $40 \mathrm{pg} / \mathrm{mL}$ in both VilCreERT2 and GcgRA ${ }^{\Delta V i l C r e E R T 2}$ mouse models (Figure 2, A-D). However, only glucose and peptone administration significantly increased insulin levels over baseline (Figure 2, E and F) and this increase was much greater with glucose than peptone. Although 2 different caloric loads of lipid (olive oil vs. intralipid) also mark- 

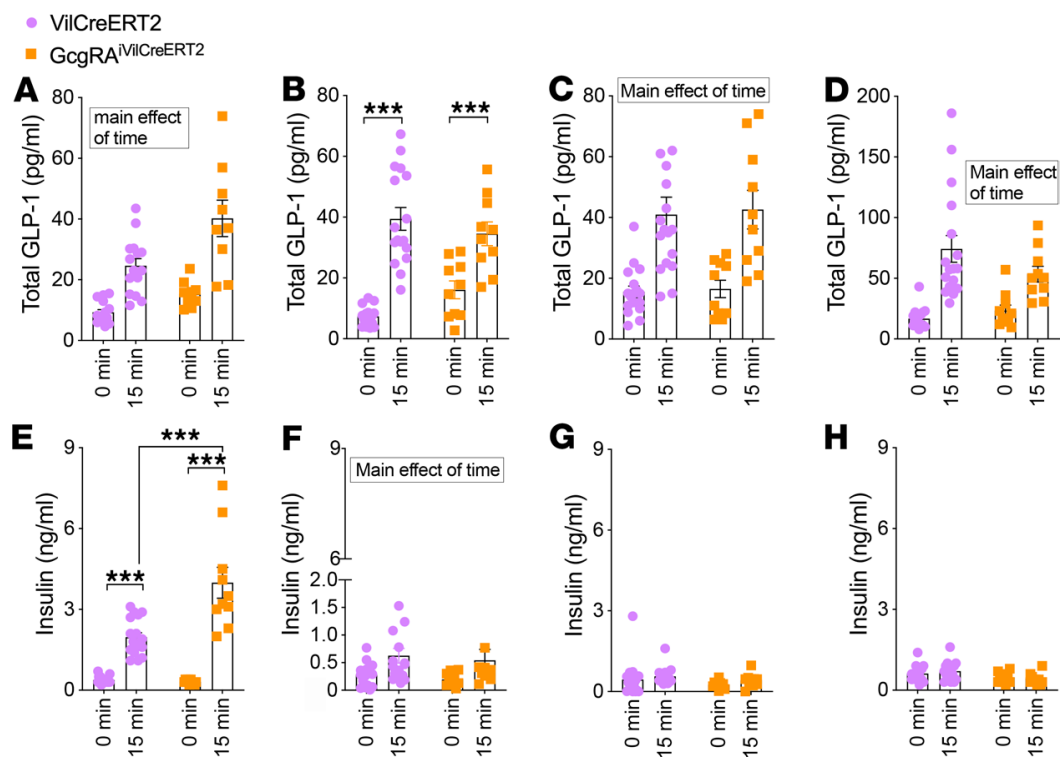

Figure 2. Intestinal GLP-1 secretion is stimulated by various nutrients, but is not insulinotropic. GLP-1 levels in response to equicaloric ( $0.34 \mathrm{Kcal}$ ) doses of $(\mathbf{A})$ glucose (main effect of time); (B) peptone; ${ }^{* * *} P<0.001$ (time $\times$ genotype), (C) intralipid (main effect of time), or (D) $1.62 \mathrm{Kcal}$ of olive oil (main effect of time). Insulin levels in response to (E) glucose; ${ }^{* *} P<0.01$ (time $\times$ genotype), (F) peptone (main effect of time), (G) intralipid, or (H) 1.62 Kcal of olive oil. All data in this figure were statistically analyzed with a 2-way ANOVA with Tukey post hoc where appropriate, were obtained from cohort 1 , each animal was tested once per condition, and are represented as Mean \pm SEM. VilCreERT2 $(n=17)$; GcgRA ${ }^{\text {AvilcreeRT2 }}(n=10)$.

edly increased intestinal GLP-1 secretion (Figure 2, C and D), neither increased insulin secretion (Figure 2, G and $\mathrm{H}$ ). The key point here is that the reactivation of Gcg in the intestine was sufficient to restore the circulating levels of total GLP-1 in response to a variety of nutrients, but it was only glucose that increases insulin.

GLP-1 secretion and GLP-1R signaling are conserved during ingestion of an HFD. Both obesity and T2DM have been reported to alter plasma GLP-1 levels, albeit with conflicting reports (22-25). To determine whether dietary-induced obesity altered the source of GLP-1 (pancreas vs. intestine) in our mouse model, we fed a $60 \%$ HFD or a chow diet to mice with developmental Gcg reactivation within the intestine (GcgRA $\left.{ }^{\Delta v i l c r e}\right)$ versus the pancreas/duodenum (GcgRA $\left.{ }^{\Delta \mathrm{Pdx} 1 \mathrm{Cre}}\right)$, to their corresponding Cre controls and to GcgRA ${ }^{\Delta \text { Null }}$ mice (see ref. 18 for the phenotype of these mice). The experimental timeline for this experiment is provided in Figure 3A. As expected, the pancreatic/duodenal contribution to circulating GLP-1 was lower compared with intestinally secreted GLP-1, but the GLP-1 response to nutrients was similar in chow versus HFD-fed mice (Figure 3, B and C). We then performed an oral glucose tolerance test (OGTT) after Ex9 or Sal administration to chow versus HFD-fed animals. We observed that the HFD substantially increased 5-hour fasting blood glucose levels (Figure 3D) and impaired glucose tolerance in all mice regardless of genotype (Figure 3, E-H). In addition, Ex9 significantly impaired glucose regulation in both chow (Figure 3I) and HFD-fed (Figure 3J) Cre control and GcgRA ${ }^{\Delta \mathrm{Pdx} I \mathrm{Cre} e}$ mice, but not in GcgRA ${ }^{\Delta \mathrm{Null}}$ or GcgRA ${ }^{\Delta \mathrm{ViC} C r e}$ mice.

Intestinal GLP-1 is not necessary for the glycemic improvements after VSG. VSG produces reliable 3- to 4-fold increases in postprandial plasma GLP-1 levels in mice $(26,27)$. We hypothesized that this large increase in postprandial GLP-1 comes from the intestine and that the degree of the increase would lead to a more prominent role for this source of GLP-1 in regulating glucose tolerance. To test this hypothesis, we performed VSG in our developmental Gcg reactivation transgenic mouse models as described previously. GcgRA ${ }^{\Delta \mathrm{Pdx} 1 \mathrm{Cre}}, \mathrm{GcgRA}^{\Delta \mathrm{Vil} C \mathrm{re}}$, GcgRA $^{\Delta N u l l}$, and the corresponding Cre control mice received sham or VSG surgery after 6 weeks on an HFD. The experimental timeline for the sham and VSG mouse cohorts is provided in Figure 4A. Gcg gene expression and pancreatic glucagon staining of the sham and VSG mouse cohorts are provided in Supplemental Figure 1 (supplemental material available online with this article; https://doi.org/10.1172/jci.insight.129452DS1) and show that Gcg expression was successfully restored in the GcgRA ${ }^{\Delta V i l c r e}$ mouse intestine and the GcgRA ${ }^{\Delta \mathrm{PDX} \text { - }}$ 1 cre mouse pancreas, respectively, whereas there was no detectable Gcg expression found in either organ in the GcgRA ${ }^{\Delta N u l l}$ mice. VSG prominently reduced body weight (BW) (Figure 4, B and C; and Supplemental Figure 2) and fat mass (Figure 4, D and E) in all mouse models compared with their corresponding sham counterparts. 
A
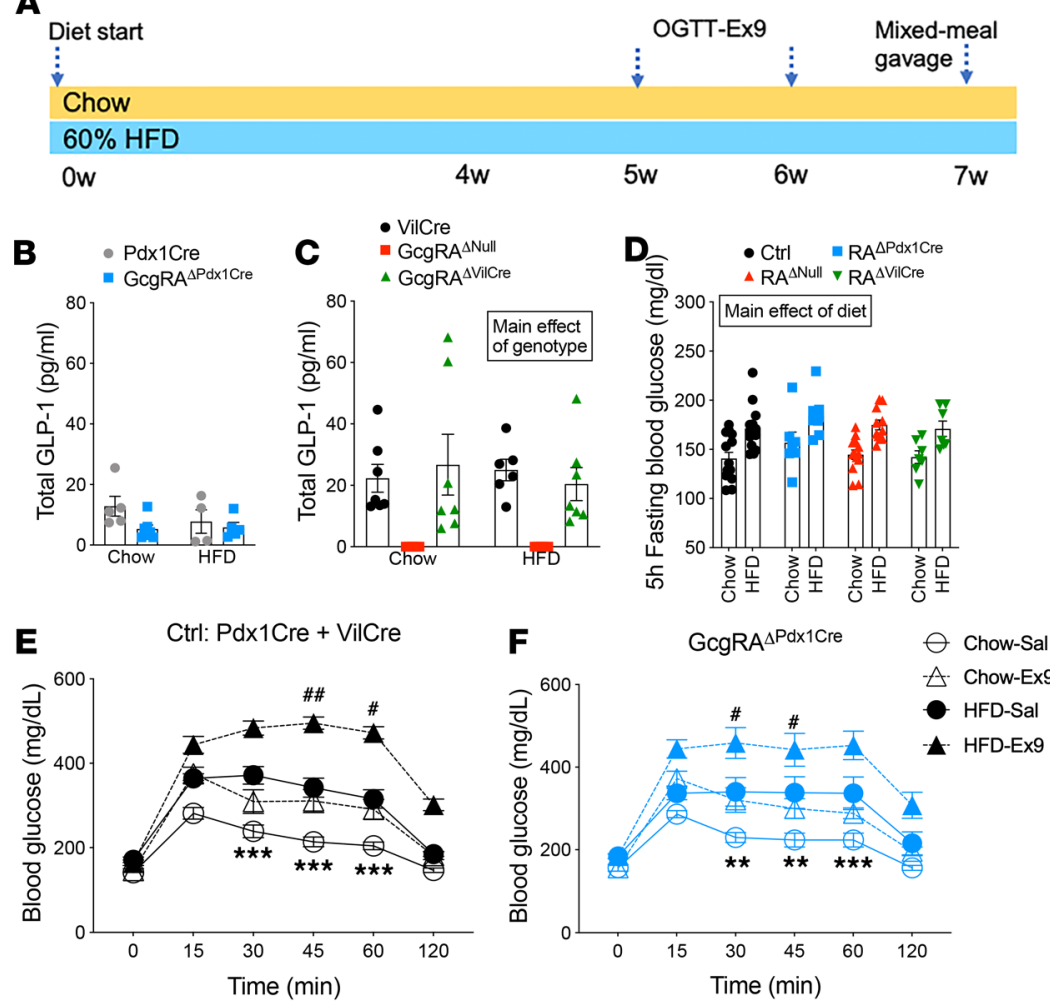

G
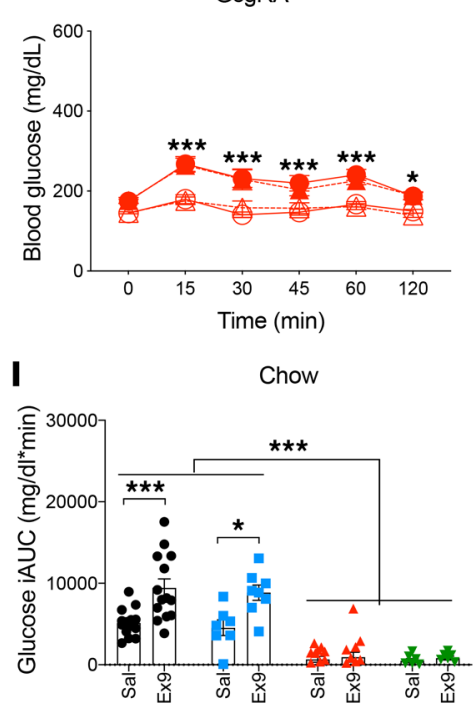

H

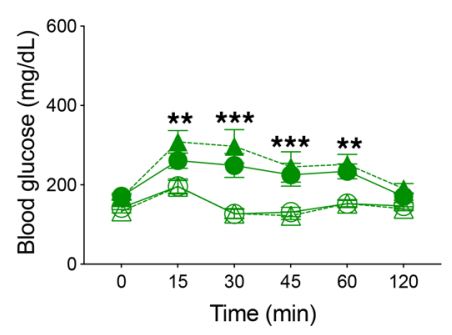

J

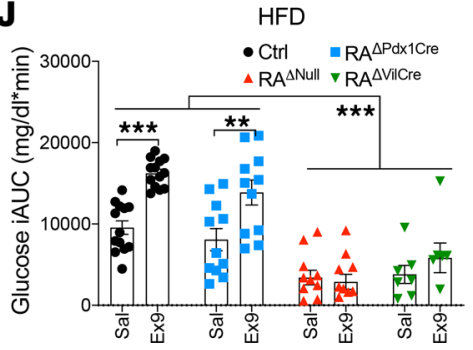

Figure 3. GLP-1 secretion and GLP-1R signaling is conserved during HFD ingestion. (A) A schematic representation of the experimental timeline for the chow versus high-fat diet (HFD) studies. (B and C) Pancreatic (B) and intestinal (C) total CLP-1 response to a liquid mixed-meal was similar between chow and HFD and was undetectable in GcgRA ${ }^{\Delta \text { Null }}$ mice (2-way ANOVA). (D) Five-hour fasting blood glucose levels were significantly greater in HFDversus chow-fed mice across all mouse genotypes (2-way ANOVA; main effect of diet). (E-H) Clucose response to an oral glucose load preceded by an i.p. injection of saline (Sal) or exendin 9-39 (Ex9) in chow- or HFD-fed control in (E) control animals (PDX1Cre and VilCre; 3-way ANOVA; time $x$ drug $x$ diet), in (F) GcgRA ${ }^{\Delta \text { Pdx } x \text { Cre }}$ (3-way ANOVA; time $x$ drug), in (G) GcgRA ${ }^{\Delta \text { Null }}$ (3-way ANOVA; time $x$ diet), and in (H) GcgRA ${ }^{\Delta V i l c r e}$ mice (3-way ANOVA; time $\times$ diet). For panels (E-H) ${ }^{*} P<0.05$; ${ }^{* *} P<0.01 ; P<0.001$ for chow versus HFD in both drug-treated groups; ${ }^{\#} P<0.05 ;{ }^{\# \#} P<0.01$ for Sal versus Ex 9 in both diet groups. Glucose incremental area under the curve (iAUC) during the oral glucose tolerance test (OGTT) in chow- (I) or HFD (J)-fed GcgRA mouse cohorts. ${ }^{*} P<0.05$; ${ }^{* *} P<0.01$; ${ }^{* *} P<0.001$ (2-way ANOVA; drug $\times$ genotype). All data were obtained from cohorts 2 and 3 , each animal was tested once per condition, and data are represented as Mean \pm SEM. OCTT data from Pdx1Cre and VilCre were combined for the Ctrl group (D, E, I, and J). Ctrl: Chow ( $n=7$ from Pdx1Cre, $n=7$ from VilCre), HFD ( $n=6$ from Pdx1Cre, $n=7$ from VilCre); GcgRA ${ }^{\Delta P d x 1 \text { ree: Chow }}$ $(n=8), \operatorname{HFD}(n=11)$; GcgRA ${ }^{\Delta \text { Null: }}$ Chow $(n=13), \operatorname{HFD}(n=11)$; GcgRA $^{\Delta \text { Villre }}$ : Chow $(n=7)$, HFD $(n=7)$.

VSG significantly increased postprandial secretion of total GLP-1 levels by approximately 4-fold compared with the sham surgery group in both the Cre control and GcgRA ${ }^{\Delta V i l C r e}$ mice (Figure 5, A and B). The sham surgery GcgRA ${ }^{\triangle \mathrm{Pdx} 1 \mathrm{Cre}}$ mice had half the levels of postprandial circulating GLP-1 compared with the sham surgery Cre control mice. After VSG, the GcgRA ${ }^{\Delta \mathrm{Pdx} 1 \mathrm{Cre}}$ mice had a 2.7-fold increase in GLP-1 compared with their sham counterparts (Figure 5A). As expected, plasma GLP-1 was not detectable in the GcgRA ${ }^{\Delta \text { Null }}$ sham or VSG mice (Figure 5B). Postprandial plasma GIP levels were markedly increased ( 2-fold) after VSG versus sham surgery (main effect of surgery; Figure 5, C and D). When analyzed independently of surgery, HFD-fed sham GcgRA ${ }^{\Delta \text { Null }}$ mice showed relatively increased GIP levels compared to HFD-fed VilCre mice (Figure 5D).

We next asked whether the 4-fold increase in postprandially secreted intestinal GLP-1 after VSG contributed to the surgery-induced improvements in glucose tolerance. First, VSG markedly reduced the 5-hour fasting blood glucose levels in all mouse models, regardless of genotype (Figure 5E). Five 
A

\begin{tabular}{lccccc} 
Diet start & Sham or VSG & OGTT-Ex9 & \multicolumn{2}{c}{ Mixed-meal } \\
$\vdots$ & $\vdots$ & $\vdots$ & $\vdots$ & gavage \\
$60 \%$ HFD & $\vdots$ & & & & \\
$-6 \mathrm{w}$ & Ow & $5 \mathrm{w}$ & $6 \mathrm{w}$ & $10 \mathrm{w}$
\end{tabular}
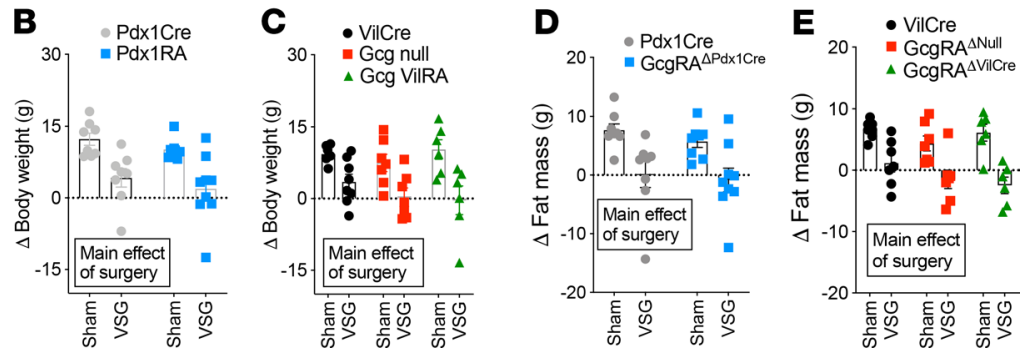

Figure 4. Gcg is not necessary in the impact of VSC on BW loss. (A) A schematic of the experimental timeline for the vertical sleeve gastrectomy (VSC) study. Body weight (BW) change from baseline versus 10 weeks ( $\triangle \mathrm{BW}$ ) after sham or VSC surgery in GcgRA ${ }^{\Delta \mathrm{Pdx} C \mathrm{Cre}}$ (B) and GcgRA ${ }^{\Delta V i l c r e}$ (C) mouse cohorts (2-way ANOVA; main effect of surgery). Fat mass changes in $\mathrm{GcgRA}^{\Delta \mathrm{Pdx} \text { Ire }}(\mathbf{D})$ and GcgRA ${ }^{\Delta v i l c r e}(\mathbf{E})$ mouse cohorts 10 weeks after surgery (2-way ANOVA main; effect of surgery). All data were obtained from cohorts 4 and 5 and are represented as Mean \pm SEM. Pdx1Cre: sham $(n=8)$, VSC $(n=8)$; GcgRA ${ }^{\Delta P d x I C r e}:$ sham ( $n=8)$, VSG $(n=9)$; VilCre: sham $(n=7)$, VSG $(n=9)$; GcgRA ${ }^{\Delta N u l l:}$ sham $(n=7)$, VSG $(n=7)$; GcgRA ${ }^{\Delta V i l(r e: ~} \operatorname{sham}(n=6)$, VSG $(n=6)$. See Supplemental Figure 2 for the linear BW changes after VSC separated by the genotype.

to 6 weeks after surgery, we performed an OGTT after administration of Ex9 or Sal. Despite being maintained on an HFD after surgery, the VSG-Cre mice showed a rapid return toward baseline glucose levels from 15 to 30 minutes after the glucose load (Figure 5F). Administration of Ex9 prevented this rapid drop in glucose (Figure 5F), indicating GLP-1R signaling mediates postprandial glycemic patterns after VSG. Similar to Cre control mice, VSG improved glycemia in GcgRA ${ }^{\triangle P d x 1 C r e}$ mice and this improvement was blocked with Ex9 pretreatment (Figure 5G). As noted previously (18), both the sham surgery GcgRA ${ }^{\Delta \text { Null }}$ and GcgRA ${ }^{\Delta V i l C r e}$ mice had improved glucose tolerance that was likely the result of the deficiency in glucagon compared with the Cre control mice. Nonetheless, we observed significant glycemic improvements after VSG in both the GcgRA ${ }^{\Delta \text { Null }}$ and GcgRA ${ }^{\Delta V i l C r e}$ mice (Figure 5 , $\mathrm{H}$ and I). However, glucose tolerance was not impaired with Ex9 treatment in either GcgRA ${ }^{\Delta \text { Null }}$ and GcgRA $^{\Delta V i l C r e}$ mice (Figure 5, J and K), indicating that intestinal Gcg is dispensable in the VSG-induced improvements in glucose tolerance.

\section{Discussion}

The investigation of GLP-1 biology over the last 3 decades has mainly focused on the intestinal source. GLP-1, which was initially found in the gut, stimulates glucose-stimulated insulin secretion when administered exogenously, and this finding led to the development of long-acting GLP-1R agonists in the treatment of T2DM. This success potentiated the hypothesis that intestinal GLP-1 is an important regulator of glucose homeostasis. However, the physiological effect of endogenous GLP-1 must be considered separately from the impact of pharmacological agonists that do not recapitulate the circulating levels or dynamics of secretion that occur with endogenous secretion (28).

Our GcgRA mouse models enabled the investigation of the role of endogenous preproglucagon products from different target organs (pancreas vs. intestine). A key finding of this work comports with the overarching model for GLP-1 as a gut hormone. In mice in which GLP-1 secretion is restored only in the intestine, various nutritional stimuli and bariatric surgery (i.e., VSG) resulted in increased levels of circulating GLP-1 that were comparable to control mice (see Figure 2). Consequently, under these conditions, the intestine is the primary source of circulating GLP-1. However, high levels of circulating GLP-1 did not necessarily result in increases in insulin levels or improved glucose homeostasis. It is important to note that GLP-1-induced regulation of insulin secretion requires normal glucose levels (6); GLP-1 does not stimulate insulin secretion under hypoglycemic conditions. In our investigation, animals were fasted for only 5 hours, providing sufficient glucose for the levels of plasma GLP-1 secreted by the intestine to induce insulin secretion if it were possible. In addition, nutrient-induced GLP-1 levels were not altered by a HFD, indicating that a HFD does not alter the relative contributions of gut versus pancreas/duodenum to circulating GLP-1 levels (see Figure 3, B and C). 
A

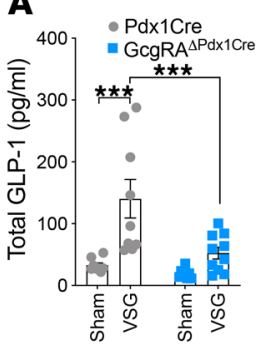

$\mathbf{E}$
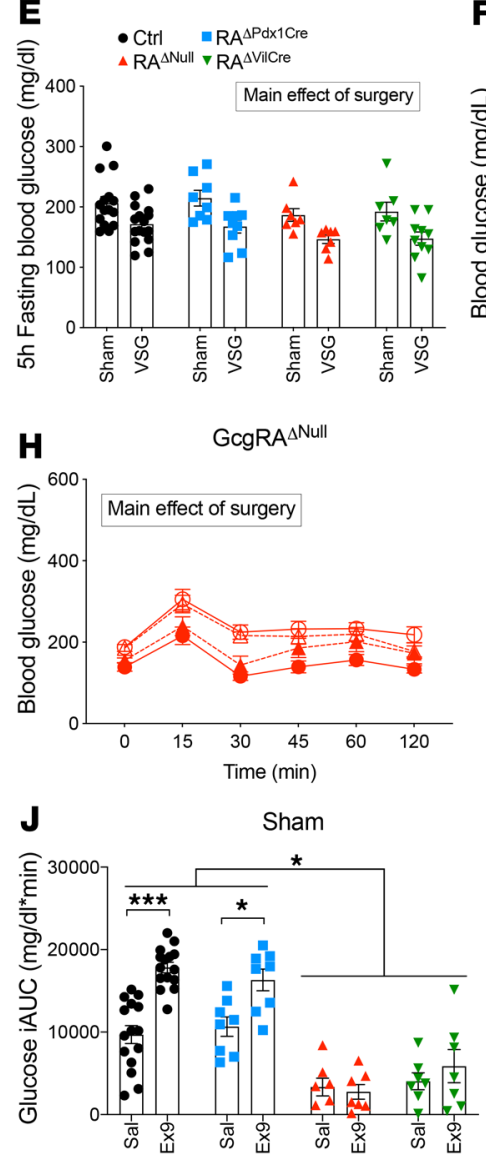

B
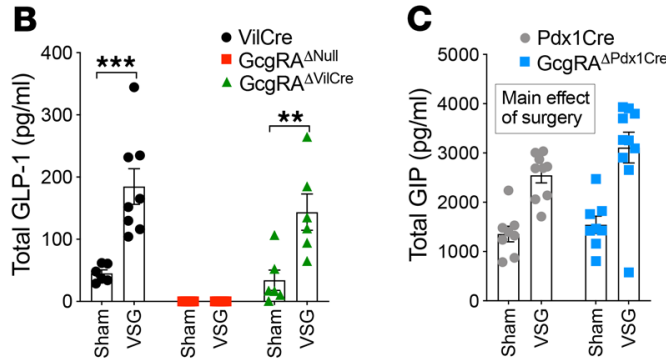

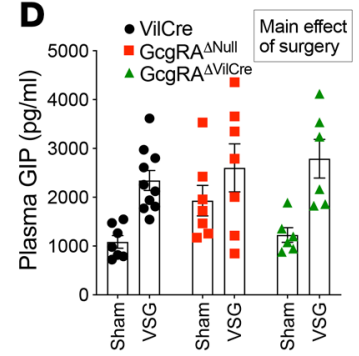

G
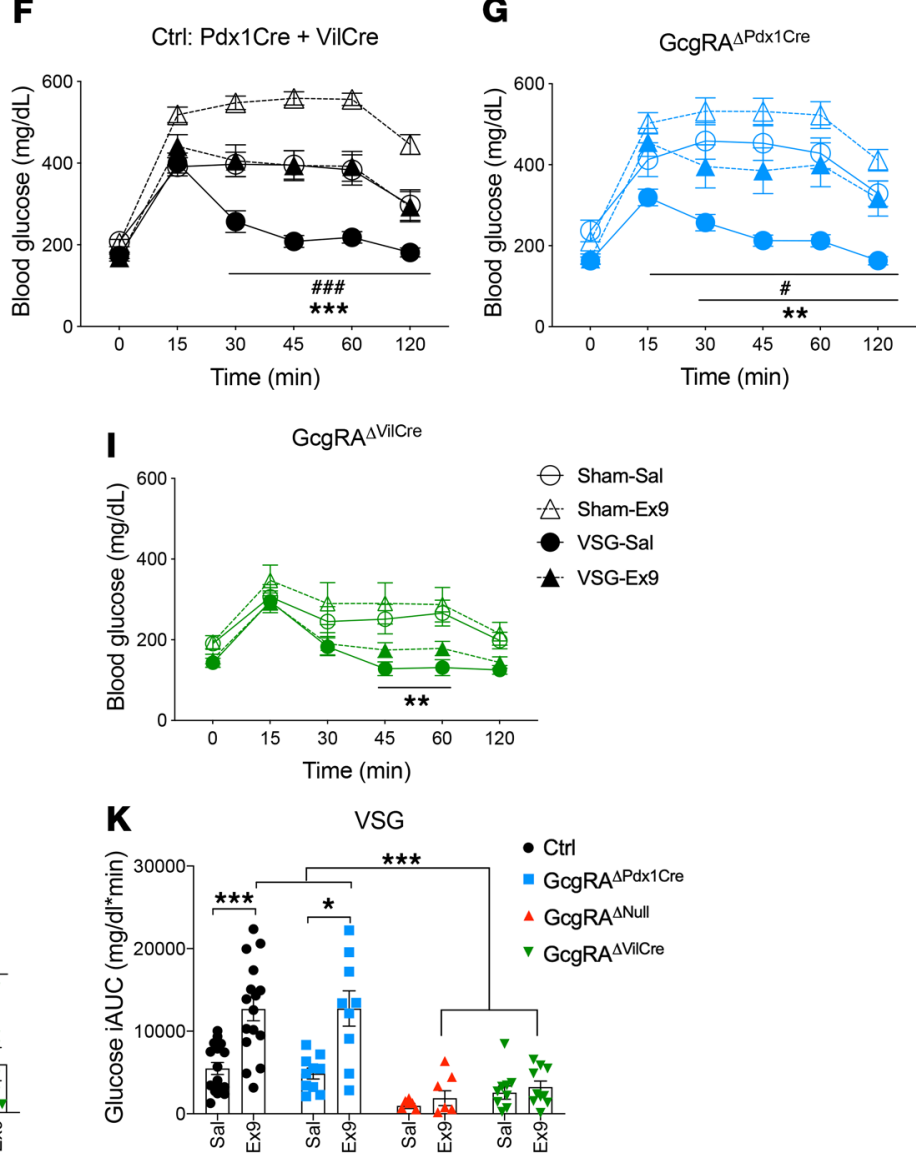

Figure 5. Increased intestinal GLP-1 secretion is not necessary in VSG-induced improvements in oral glucose tolerance. Plasma total GLP-1 levels 15 minutes after a liquid mixed nutrient gavage in sham versus vertical sleeve gastrectomy (VSG) (A) GcgRA ${ }^{\Delta P d x i c r e}$ and (B) GcgRA ${ }^{\Delta v i l c r e}$ mouse cohorts. ${ }^{* *} P<0.01$; ${ }^{* *} P<0.001$ (2-way ANOVA; genotype $\times$ surgery). Total gastric inhibitory polypeptide (GIP) response to the liquid mixed nutrient gavage in sham versus VSG (C) GcgRA ${ }^{\Delta P d x i c r e}$ and (D) GcgRA $\Delta$ Vilicre mouse cohorts (2-way ANOVA; main effect of surgery). (E) Five-hour fasting blood glucose was significantly lower in VSG versus sham animals (2-way ANOVA; main effect of surgery). Glucose response to an oral glucose load after an i.p. injection of saline (Sal) or exendin 9-39 (Ex9) in sham or VSG (F) control (Ctrl; ${ }^{* * *} P<0.001$ for sham vs. VSG in each drug-treated group [3-way ANOVA; time

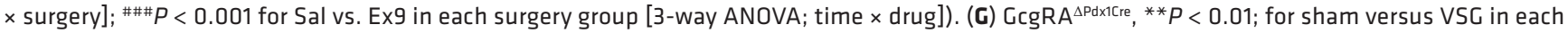
drug-treated group (3-way ANOVA; time $\times$ surgery); ${ }^{\#} P<0.05$; for Sal versus Ex9 in each surgery group (3-way ANOVA; time $\times$ drug). (H) GcgRA ${ }^{\Delta N u l l}$ (3-way ANOVA; main effect of surgery), and (I) GcgRA ${ }^{\Delta v i l r e}$ mice. ${ }^{* *} P<0.01$; for sham versus VSC in each drug-treated group (2-way ANOVA; time $\times$ surgery). (J) Glucose incremental area under the curve (iAUC) during the oral glucose tolerance test (OGTT) in sham- (J) or VSG-treated (K) GcgRA mouse cohorts. ${ }^{*} P<0.05$; ${ }^{* *} P<0.001$ (3-way ANOVA; genotype $\times$ drug). All data were obtained from cohorts 4 and 5 , all animals were tested once per condition, and data are represented as Mean \pm SEM. OGTT data from Pdx1Cre and VilCre were combined as control (E, F, J, and K). Control: sham ( $n$ $=8$ from Pdx1Cre, $n=7$ from VilCre), VSG ( $n=8$ from Pdx1Cre, $n=9$ from VilCre); GcgRA ${ }^{\Delta P d x i c r e}$ sham $(n=8)$, VSG $(n=9) ;$ GcgRA ${ }^{\Delta N u l l:}$ sham ( $\left.n=7\right)$, VSG $(n=7)$; GcgRA ${ }^{\Delta v i l c r e: ~} \operatorname{sham}(n=6), \operatorname{VSC}(n=6)$.

Although the intestine is clearly the important source of circulating GLP-1 under various nutrient conditions, a key question is whether gut-derived GLP-1 is an important regulator of insulin secretion and postprandial glucose homeostasis. Our previous work called into question this hypothesis by treating these various mouse models with Ex9 to block GLP-1 receptor signaling. Ex9 was able to disrupt normal 
glucose regulation but only in mice in which endogenous secretion had been restored in the pancreas (18). Our current work directly replicated and extended those results. Once again, under normal chow-fed conditions, Ex9 had no impact in mice that do not make any preproglucagon products (including GLP-1); this finding indicated that Ex9 exerts its effects as a competitive antagonist to GLP-1 (see Figure 3G). Ex9 was also ineffective in mice in which circulating GLP-1 levels were restored by reactivating endogenous intestinal Gcg expression. However, Ex9 was effective at impairing glucose homeostasis in mice when GLP-1 production was restored selectively in the pancreas. The exact same pattern of results occurred after these various mouse models were exposed to a HFD, indicating that greatly altering the diet does not alter the relative contributions of gut versus pancreas-derived GLP-1 to normal glucose regulation.

We believe GLP-1 secreted from the pancreas acts in a paracrine, rather than endocrine, manner to regulate insulin and consequently glucose homeostasis. This is supported by our data indicating that the primary source of circulating GLP-1 comes from the intestine, not the pancreas (see previous discussion). Although controversial, $\alpha$ cells have been found to express the prohormone convertase $1 / 3(\mathrm{PC} 1 / 3)$ that is required for posttranslational processing of proglucagon to GLP-1 (and other peptides including GLP-2) $(17,29)$, and pancreatic levels of active GLP-1 increase with nutrient stimulation $(17,30)$. However, recent perifusion experiments found that glucagon also acts on $\beta$ cell GLP-1R, and not glucagon receptors, to regulate insulin secretion in response to amino acids (31-33). Together with our in vivo data, these data indicate that a major function of proglucagon peptides is to regulate insulin secretion in a paracrine fashion and this signaling is important in regulation of glucose tolerance.

Several bariatric surgeries, including VSG, result in pronounced increases in GLP-1 secretion in rodents and in humans (reviewed in ref. 34). Wide-ranging debate exists regarding the degree to which this increase contributes to the potent metabolic effects of these surgeries $(35,36)$. It is reasonable to hypothesize that the dramatic increase in GLP-1 secretion could come from the intestine and that such large increases in circulating levels may make gut-derived GLP-1 a more important contributor to glucose homeostasis than under normal conditions. Indeed, it is the case that most of the increased circulating levels of GLP-1 after VSG are derived primarily from the intestine (see Figure 5, A and B). However, we find that the potent effect of VSG to alter body fat and lower fasting glucose levels does not rely on GLP-1 or other Gcg products (see Figure 4, B and C). Importantly, blocking endogenous GLP-1 signaling with Ex9 impairs glucose regulation similarly in sham versus VSG mice, an effect that is absent both in mice totally devoid of GLP-1 production and in those with restored circulating GLP-1 via reactivation in the gut. When GLP-1 production is restored to the pancreas in VSG-treated mice, once again Ex9 impairs postprandial glucose regulation. The important point here is that even after VSG, the critical source of the preproglucagon products that contribute to normal glucose regulation is the pancreas.

Previous data have suggested that whole-body GLP-1R signaling is not necessary for either VSG (26) or Roux-en-Y gastric bypass (RYGB) (37) to induce weight loss and improve glucose tolerance. However, 2 recent studies using 2 different models of inducible $\beta$ cell-specific GLP-1R-KO mice demonstrated completely opposite effects; one model demonstrated that these receptors are necessary (30), whereas the other model demonstrated that these receptors are not necessary (38) for the changes in glucose homeostasis after VSG. Whether the minor differences in HFD type and timing of exposure, the mouse models used, or some unknown factor may contribute either independently, or in combination, to the divergent results is unknown. It is also possible that a combination of factors is important in the metabolic success of VSG as has been demonstrated previously (39). Therefore, we cautiously interpret our data to say that pancreatic GLP-1 is important for postprandial glycemic excursions with VSG, but whether this signal, in and of itself, is critical for the resolution of diabetes mellitus with VSG cannot be determined from these mouse models.

The lack of glucagon in the GcgRA ${ }^{\Delta \text { null }}$ and GcgRA ${ }^{\Delta V i l C r e}$ mice, independent of surgery, improved glucose tolerance (sham null and intestinally reactivated mice vs. sham controls). Despite this already improved glucose tolerance, these mice still had an additional improvement in glucose tolerance with VSG. These data highlight the Gcg-independent factors that may be regulating the improvements in glucose homeostasis after surgery, including potential compensatory increases in other gut peptides (i.e., GIP, PYY, etc.), reduced BW, and/or improved insulin-dependent or -independent glucose disposal. In terms of limitations of this study, we were unable to assess whether the insulin responses differed between the genetic models after VSG because of the limited blood volume in mice and the fact that we prioritized the assessment of gut peptides. Finally, we cannot exclude the possibility that the paracrine action of GLP-1 is more import- 
ant in mice than it is humans. GLP-1 and PC1/3 are found in the pancreas of humans, and the anatomy of the islet in humans ( $\alpha$ cells are dispersed throughout rather than around the perimeter) may favor a paracrine role for GLP-1 in the islet $(17,29,40)$. Nevertheless, more research is needed to confirm the relative role of pancreatic versus intestinal GLP-1 in regulating glucose homeostasis in humans.

We realize that these data challenge the widely held hypotheses about the role of GLP-1 as a gut hormone that regulates insulin and glucose. If not regulation of glucose homeostasis, then what is the physiological role(s) on intestinally secreted GLP-1? GLP-1R expression is found in intestinal epithelial lymphocytes and has been found to regulate the enteric immune response (41). Lipopolysaccharide, an endotoxin produced by gram-negative bacteria in the intestine, stimulates the secretion of GLP-1 (42) in an interleukin-6-dependent manner (43), and patients with sepsis also have increased levels of plasma GLP-1 (42). These data directly link plasma GLP-1 to immune responses to infection and to physiological stress. However, whether GLP-1, per se, mediates the immune response or whether the increase is simply a marker of intestinal stress remains unclear.

Here, we find that various nutrients stimulate intestinal GLP-1 secretion, but the function of this source of GLP-1 is likely not regulation of insulin secretion and consequently glucose homeostasis. In fact, even with the 4-fold increases in intestinal GLP-1 secretion after VSG, intestinal GLP-1 is still not an important regulator of postprandial glucose levels. Thus, our data indicate that, under various dietary conditions and after VSG, it is pancreatic, not intestinal GLP-1 that is the primary source of endogenous GLP-1 that regulates meal-induced glucose tolerance. These data further support a paracrine role for pancreatic preproglucagon products in insulin secretion.

\section{Methods}

Animals. Male mice were single-housed under a 12-hour light/dark cycle with ad libitum access to water and standard chow (Envigo Teklad; catalog 7012) or 60\% HFD; Research Diet; catalog D12492). The animal room was maintained at $25^{\circ} \mathrm{C}$ with $50 \%-60 \%$ humidity. All studies were performed in animals 8-16 weeks of age with age-matched littermate $\mathrm{Cre}$ control mice and all mice were euthanized with a $\mathrm{CO}_{2}$ inhalation. A total 5 cohorts of male transgenic mouse models with mixed backgrounds (i.e., no backcrosses were performed in these mice) are used in this study (Supplemental Table 1).

Our previous work determined that developmental reactivation of intestinally derived Gcg restored baseline and postprandial levels of GLP-1, but this source of GLP-1 was not necessary for glucose homeostasis (18). To determine whether reactivation of intestinally derived Gcg in adulthood would reveal an important contribution of this source of GLP-1 to glucose homeostasis, a previously validated Gcg-null mouse model $\left(G_{c g R A}{ }^{\Delta \text { Null }}\right)$ that with a LoxP-flanked transcriptional blocking cassette inserted into the Gcg gene (18) was crossed with a tamoxifen-inducible VilCre (VilCre-ER ${ }^{\mathrm{T} 2}$ ) mouse (21). The resulting $\operatorname{GcgRA}^{\Delta V i l C r e E R 2}(n=10)$ and littermate control $(n=17)$ mice were all administered tamoxifen (Sigma-Aldrich; catalog T5648) by i.p. injection every other day $(120 \mathrm{mg} / \mathrm{Kg} /$ day $\times 3$ administrations). After the last injection, the mice were acclimated for 7 days before any further studies.

In separate studies, we crossed the GcgRA ${ }^{\Delta \text { Null }}$ mice with the developmental VilCre (44) (stock no. 004586) or Pdx1Cre (45) (stock no. 014647; both Cre mouse lines purchased from the Jackson Laboratory) to reactivate endogenous Gcg expression within the intestine $\left(\mathrm{GcgRA}^{\Delta \mathrm{VilCr}}\right)$ or pancreas/duodenum $\left(G_{c g R A}{ }^{\Delta \mathrm{Pdx} 1 \mathrm{Cr} e}\right)$, respectively. These $\mathrm{GcgRA}^{\Delta \mathrm{Pdx} 1 \mathrm{Cre}}$ and $\mathrm{GcgRA}^{\Delta \mathrm{Vil} \text { re }}$ transgenic mouse models were validated previously (18). These mice were metabolically phenotyped after 7 weeks on chow or HFD (Cohorts 2 and 3) or were placed on an HFD for 6 weeks and then had sham or VSG surgery (Cohorts 4 and 5). Supplemental Table 1 describes these cohorts in detail.

Nutrient stimulation. On separate occasions, each spaced by 10 days, mice were fasted for 5 hours and baseline blood was taken from the tail vein and collected in EDTA-coated microtubes containing a DPP4 inhibitor (MilliporeSigma). The mice were then gavaged with 50\% dextrose solution (volume $200 \mathrm{~L}$; Hospira Inc.), 20\% intralipid (volume $170 \mu \mathrm{L}$; Fresenius Kabi), or peptone (40\% (w/v) solution (volume $321 \mu \mathrm{L}$; Primatone RL, Sigma-Aldrich; catalog P4963); each nutrient equating to $0.34 \mathrm{Kcal}$. In another study, a higher caloric load of extra virgin olive oil (200 L; $1.62 \mathrm{Kcal})$ was orally administered. Fifteen minutes after the nutrient gavage, blood was taken from the tail vein $(<40 \mu \mathrm{L}$ total blood), centrifuged, and subsequently analyzed for insulin and GLP-1 levels.

In all cohorts, we performed an OGTT following a 5-hour fast. A potent GLP-1R antagonist, Ex9 (Bachem), or saline was administered i.p. to each mouse 15 minutes before an oral glucose gavage $(2 \mathrm{~g} /$ 
$\mathrm{Kg}$ of $50 \%$ dextrose). Blood collected from the tail vein was assessed for glucose levels using a hand-held glucometer (Accu-check Aviva Plus; Roche Diagnostics) at 0, 15, 30, 45, 60, and 120 minutes after administration of glucose. All animals were studied in a cross-over design to determine the impact of saline versus Ex9 on glucose tolerance and experiments were spaced by at least 1 week.

Cohorts 3 and 4 were maintained on chow or $60 \%$ HFD for 7 weeks. The mice were fasted for 5 hours prior to an oral administration of liquid mixed-meal (volume $200 \mu \mathrm{L}$; Ensure plus spiked with a 30-mg dextrose). At 15 minutes after the oral meal ingestion, blood was taken from the tail vein and collected in EDTA-coated microtubes containing a DPP4 inhibitor.

Bariatric surgery. Cohorts 4 and 5 were maintained on $60 \%$ HFD for 6 weeks to induce obesity, were matched for body fat within a genotype, and then received sham or VSG surgery as described previously (46, 47). Briefly, mice were anesthetized using isoflurane, and a small laparotomy incision was made in the abdominal wall. The VSG procedure involved the excision of the lateral $80 \%$ of the stomach along the greater curvature using an ETS 35-mm staple gun (Ethicon Endo-Surgery), leaving a vertical gastric sleeve that is continuous with the esophagus and pylorus. The sham surgery involved the application of gentle pressure on the stomach with blunt forceps. During the first 3 days of the postoperative period, the animals were fed Osmolite 1.0 Cal liquid diet (Abbott Nutrition) and then were returned to the $60 \%$ HFD. BW was monitored for 10 weeks after surgery. Body composition was measured using an EchoMRI (Echo Medical Systems) before and 10 weeks postoperatively. Five to 6 weeks after surgery, we performed an OGTT with an i.p. administration of saline or Ex9 as described previously. At 10 weeks after surgery, the mice were fasted for 5 hours prior to an oral administration of liquid mixed-meal (volume $200 \mu \mathrm{L}$; Ensure plus spiked with a 30-mg dextrose). At 15 minutes after the oral meal ingestion, the mice were euthanized with a $\mathrm{CO}_{2}$ inhalation and blood was taken immediately through a cardiac puncture and collected in EDTA-coated microtubes containing a DPP4 inhibitor and an aprotinin. Cohort 4 consisted with Pdx1Cre: sham $(n=8)$, VSG $(n=8)$; GcgRA ${ }^{\Delta \mathrm{Pdx} 1 \mathrm{Cre}}$ : sham $(n=8)$, VSG $(n=9)$. Cohort 5 consisted with VilCre: sham ( $n=7)$, VSG ( $n=9)$; GcgRA ${ }^{\Delta \text { Null: }}$ sham $(n=7)$, VSG $(n=7) ; \operatorname{GcgRA}^{\Delta V i l c r e}: \operatorname{sham}(n=6)$, VSG $(n=6)$.

ELISA. Total GLP-1 (MesoScale Discovery; catalog K150JVC) was assayed using a sandwich ELISA assay kit, whereas total GIP (MilliporeSigma; catalog EZRMGIP-55K) and insulin (Crystal Chem; catalog 90080) were assayed using a standard ELISA assay kit. All assays were performed according to the manufacturer's instructions.

Gene expression analysis. Mouse hindbrain, whole pancreas, and epithelial scrapes from the duodenum, jejunum, and ileum were immediately homogenized into Trizol (Thermo Fisher Scientific) using a Tissuelyser II (Qiagen). Total RNA was extracted from the samples using Purelink RNA mini kit (Thermo Fisher Scientific). The quality and concentration of the isolated total RNA were determined using a Nanodrop spectrophotometer (Thermo Fisher Scientific). cDNA was hybridized from $1 \mu \mathrm{g}$ of each total RNA sample using an iscript cDNA hybridization kit (Bio-Rad). Taqman gene expression assay was performed using StepOne Plus real-time qPCR system and the following Taqman gene probes were used: Mouse $B 2 \mathrm{~m}$ (Mm00437762_m1) and mouse Gcg (Mm01269054_m1).

Pancreatic fluorescence immunostaining. Mouse pancreata from sham and VSG mice within the different genotypes (control, null, intestinal, and pancreatic reactivated mice; $n=4$ /group) were paraffin-embedded and sectioned onto slides by the University of Michigan In-Vivo Animal Core (IVAC). Paraffin was removed using Citrisolv (VWR). For double immunofluorescence staining, the slides were incubated overnight at $4^{\circ} \mathrm{C}$ with each primary antibody: rabbit anti-glucagon (Abcam, catalog ab92517) and chicken anti-GFP (Abcam, catalog ab13970). The corresponding secondary antibodies conjugated to Alexa Fluor dyes were as follows: donkey anti-chicken FITC (Thermo Fisher Scientific, catalog SA1-72000) and donkey anti-rabbit 594 (Thermo Fisher Scientific, catalog A21207). The slides were mounted in an anti-fade fluorescence mounting medium containing DAPI (Vectashield with DAPI, Vector Laboratories, catalog H-1200). Fluorescent images were obtained using an Olympus IX73 fluorescence microscopy system (Olympus) and were analyzed using Olympus cellSens imaging software (Olympus).

Statistics. All data were analyzed using Graphpad Prism 8 software or Statistica (TIBCO Software). Data were first analyzed to determine significant main effects and interactions between independent variables (genotype, surgery, diet, drug, and/or time). If there were no significant interactions with time but instead significant main effects, this was then indicated within a text box on the figure. Significant interactions were analyzed with a Tukey's post hoc to determine where significant differences lie. Statistical significance was set at $P<0.05$ for all analyses. Data are presented as mean \pm SEM. 
Study approval. All animal experiments were performed according to an approved protocol by the Institutional Animal Care and Use Committee (IACUC) at the University of Michigan and we followed protocols outlined in the National Institutes of Health $(\mathrm{NIH})$ guide for the care and use of laboratory animals (NIH Publications No. 8023, revised 1978).

\section{Author contributions}

KSK, CRH, LW, and IJM were responsible for executing the experiments. KSK, RJS, and DAS were responsible for the design of experiments. KSK, RJS, and DAS were responsible for analysis and interpretation of data and drafting of the manuscript. DAS provided final approval of the submitted manuscript.

\section{Acknowledgments}

The authors thank the surgeons for conducting mouse VSG (Alfor Lewis, Andriy Myronovych, Mouhamadoul Toure, and Diana Farris) and Kelli Rule for the technical assistance in mouse breeding, and the In-Vivo Animal Core (IVAC) at UM for tissue processing. This work is supported in part by NIH Awards, DK082480 (DAS), DK107652 (RJS), and DK093848 (RJS), and by an Early Career Award from the Obesity Society (KSK). This work is also supported in part by NIH-funded Michigan Nutrition and Obesity Research Center (MNORC), DK089503 (RJS).

Address correspondence to: Darleen A. Sandoval, Department of Surgery, University of Michigan, 2800 Plymouth Road, NCRC Building 26-341N, Ann Arbor, Michigan 48109-2800, USA. Phone: 734.764.6258; Email: darleens@med.umich.edu.

1. Mcintyre N, Holdsworth CD, Turner DS. New interpretation of oral glucose tolerance. Lancet. 1964;2(7349):20-21

2. Elrick H, Stimmler L, Hlad CJ, Arai Y. Plasma insulin response to oral and intravenous glucose administration. J Clin Endocrinol Metab. 1964;24:1076-1082.

3. Unger RH, Ohneda A, Valverde I, Eisentraut AM, Exton J. Characterization of the responses of circulating glucagon-like immunoreactivity to intraduodenal and intravenous administration of glucose. J Clin Invest. 1968;47(1):48-65.

4. Dupre J, Ross SA, Watson D, Brown JC. Stimulation of insulin secretion by gastric inhibitory polypeptide in man. J Clin Endocrinol Metab. 1973;37(5):826-828.

5. Drucker DJ, Philippe J, Mojsov S, Chick WL, Habener JF. Glucagon-like peptide I stimulates insulin gene expression and increases cyclic AMP levels in a rat islet cell line. Proc Natl Acad Sci U S A. 1987;84(10):3434-3438.

6. Nauck MA, et al. Effects of glucagon-like peptide 1 on counterregulatory hormone responses, cognitive functions, and insulin secretion during hyperinsulinemic, stepped hypoglycemic clamp experiments in healthy volunteers. J Clin Endocrinol Metab. 2002;87(3):1239-1246.

7. Holst JJ, Orskov C, Nielsen OV, Schwartz TW. Truncated glucagon-like peptide I, an insulin-releasing hormone from the distal gut. FEBS Lett. 1987;211(2):169-174.

8. Krarup T, Saurbrey N, Moody AJ, Kühl C, Madsbad S. Effect of porcine gastric inhibitory polypeptide on beta-cell function in type I and type II diabetes mellitus. Metab Clin Exp. 1987;36(7):677-682.

9. Kim KS, Jang HJ. Medicinal plants qua glucagon-like peptide-1 secretagogue via intestinal nutrient sensors. Evid Based Complement Alternat Med. 2015;2015:171742.

10. Gribble FM, Meek CL, Reimann F. Targeted intestinal delivery of incretin secretagogues - towards new diabetes and obesity therapies. Peptides. 2018;100:68-74.

11. Ali M, El Chaar M, Ghiassi S, Rogers AM, American Society for Metabolic Bariatric Surgery Clinical Issues Committee. American Society for Metabolic and Bariatric Surgery updated position statement on sleeve gastrectomy as a bariatric procedure. Surg Obes Relat Dis. 2017;13(10):1652-1657.

12. Welbourn R, et al. Bariatric Surgery Worldwide: Baseline Demographic Description and One-Year Outcomes from the Fourth IFSO Global Registry Report 2018. Obes Surg. 2019;29(3):782-795.

13. Chambers AP, et al. Weight-independent changes in blood glucose homeostasis after gastric bypass or vertical sleeve gastrectomy in rats. Gastroenterology. 2011;141(3):950-958.

14. Williams DL, Baskin DG, Schwartz MW. Evidence that intestinal glucagon-like peptide-1 plays a physiological role in satiety. Endocrinology. 2009;150(4):1680-1687.

15. Sandoval DA, D'Alessio DA. Physiology of proglucagon peptides: role of glucagon and GLP-1 in health and disease. Physiol Rev. 2015;95(2):513-548.

16. Gaykema RP, et al. Activation of murine pre-proglucagon-producing neurons reduces food intake and body weight. J Clin Invest. 2017;127(3):1031-1045.

17. Marchetti P, et al. A local glucagon-like peptide 1 (GLP-1) system in human pancreatic islets. Diabetologia. 2012;55(12):3262-3272.

18. Chambers AP, et al. The role of pancreatic preproglucagon in glucose homeostasis in mice. Cell Metab. 2017;25(4):927-934.e3.

19. Bodnaruc AM, Prud'homme D, Blanchet R, Giroux I. Nutritional modulation of endogenous glucagon-like peptide-1 secretion: a review. Nutr Metab (Lond). 2016;13:92.

20. Steinert RE, Feinle-Bisset C, Asarian L, Horowitz M, Beglinger C, Geary N. Ghrelin, CCK, GLP-1, and PYY(3-36): secretory controls and physiological roles in eating and glycemia in health, obesity, and after RYGB. Physiol Rev. 2017;97(1):411-463. 
21. el Marjou F, et al. Tissue-specific and inducible Cre-mediated recombination in the gut epithelium. Genesis. 2004;39(3):186-193.

22. Richards $\mathrm{P}$, et al. High fat diet impairs the function of glucagon-like peptide-1 producing L-cells. Peptides. 2016;77:21-27.

23. Wang F, et al. Chronic high-fat feeding increases GIP and GLP-1 secretion without altering body weight. Am J Physiol Gastrointest Liver Physiol. 2015;309(10):G807-G815.

24. Theodorakis MJ, et al. Human duodenal enteroendocrine cells: source of both incretin peptides, GLP-1 and GIP. Am J Physiol Endocrinol Metab. 2006;290(3):E550-E559.

25. Muscelli E, et al. Separate impact of obesity and glucose tolerance on the incretin effect in normal subjects and type 2 diabetic patients. Diabetes. 2008;57(5):1340-1348.

26. Wilson-Pérez HE, et al. Vertical sleeve gastrectomy is effective in two genetic mouse models of glucagon-like Peptide 1 receptor deficiency. Diabetes. 2013;62(7):2380-2385.

27. Hutch CR, et al. Oea signaling pathways and the metabolic benefits of vertical sleeve gastrectomy [published online ahead of print November 20, 2018]. Ann Surg. https://doi.org/10.1097/SLA.0000000000003093.

28. Gao W, Jusko WJ. Target-mediated pharmacokinetic and pharmacodynamic model of exendin-4 in rats, monkeys, and humans. Drug Metab Dispos. 2012;40(5):990-997.

29. Traub S, et al. Pancreatic $\alpha$ cell-derived glucagon-related peptides are required for $\beta$ cell adaptation and glucose homeostasis. Cell Rep. 2017;18(13):3192-3203.

30. Garibay D, et al. $\beta$-Cell glucagon-like peptide-1 receptor contributes to improved glucose tolerance after vertical sleeve gastrectomy. Endocrinology. 2016;157(9):3405-3409.

31. Capozzi ME, et al. $\beta$ Cell tone is defined by proglucagon peptides through cAMP signaling. JCI Insight. 2019;4(5):126742.

32. Svendsen B, et al. Insulin secretion depends on intra-islet glucagon signaling. Cell Rep. 2018;25(5):1127-1134.e2.

33. Zhu L, et al. Intra-islet glucagon signaling is critical for maintaining glucose homeostasis. JCI Insight. 2019;4(10):127994.

34. Kim KS, Sandoval DA. Endocrine function after bariatric surgery. Compr Physiol. 2017;7(3):783-798.

35. Nannipieri M, et al. Roux-en-Y gastric bypass and sleeve gastrectomy: mechanisms of diabetes remission and role of gut hormones. J Clin Endocrinol Metab. 2013;98(11):4391-4399.

36. Larraufie P, et al. Important role of the GLP-1 axis for glucose homeostasis after bariatric surgery. Cell Rep. 2019;26(6):1399-1408.e6.

37. Mokadem M, Zechner JF, Margolskee RF, Drucker DJ, Aguirre V. Effects of Roux-en-Y gastric bypass on energy and glucose homeostasis are preserved in two mouse models of functional glucagon-like peptide-1 deficiency. Mol Metab. 2014;3(2):191-201.

38. Douros JD, et al. Enhanced glucose control following vertical sleeve gastrectomy does not require a $\beta$-cell glucagon-like peptide 1 receptor. Diabetes. 2018;67(8):1504-1511.

39. Svane MS, et al. Postprandial nutrient handling and gastrointestinal hormone secretion after Roux-en-Y gastric bypass vs sleeve gastrectomy. Gastroenterology. 2019;156(6):1627-1641.e1.

40. Whalley NM, Pritchard LE, Smith DM, White A. Processing of proglucagon to GLP-1 in pancreatic $\alpha$-cells: is this a paracrine mechanism enabling GLP-1 to act on $\beta$-cells? J Endocrinol. 2011;211(1):99-106.

41. Yusta B, et al. GLP-1R agonists modulate enteric immune responses through the intestinal intraepithelial lymphocyte GLP-1R. Diabetes. 2015;64(7):2537-2549.

42. Kahles F, et al. GLP-1 secretion is increased by inflammatory stimuli in an IL-6-dependent manner, leading to hyperinsulinemia and blood glucose lowering. Diabetes. 2014;63(10):3221-3229.

43. Ellingsgaard $H$, et al. Interleukin- 6 enhances insulin secretion by increasing glucagon-like peptide-1 secretion from $\mathrm{L}$ cells and alpha cells. Nat Med. 2011;17(11):1481-1489.

44. Madison BB, Dunbar L, Qiao XT, Braunstein K, Braunstein E, Gumucio DL. Cis elements of the villin gene control expression in restricted domains of the vertical (crypt) and horizontal (duodenum, cecum) axes of the intestine. J Biol Chem. 2002;277(36):33275-33283.

45. Hingorani SR, et al. Preinvasive and invasive ductal pancreatic cancer and its early detection in the mouse. Cancer Cell. 2003;4(6):437-450.

46. Chambers AP, et al. The effects of vertical sleeve gastrectomy in rodents are ghrelin independent. Gastroenterology. 2013;144(1):50-52.e5

47. Patel A, Yusta B, Matthews D, Charron MJ, Seeley RJ, Drucker DJ. GLP-2 receptor signaling controls circulating bile acid levels but not glucose homeostasis in $\mathrm{Gcgr}^{-1-}$ mice and is dispensable for the metabolic benefits ensuing after vertical sleeve gastrectomy. Mol Metab. 2018;16:45-54. 